\title{
Resistance nailed
}

A series of in vitro, genomic, ecological and epidemiological studies has pinpointed gene mutations in the malaria parasite Plasmodium falciparum that play a key part in resistance to artemisinin-based antimalarial drugs. SEE ARTICLE P.50

\section{CHRISTOPHER V. PLOWE}

A fragile consensus that the global eradication of malaria is possible, and prospects for eventually achieving this audacious goal, are being threatened by the emergence in southeast Asia of parasite resistance to the drug artemisinin and its derivatives. On page 50 of this issue, Ariey et al. ${ }^{1}$ report welcome news: they have identified a molecular marker of artemisininresistant malaria that can be used to map resistance and guide efforts to eliminate it.

Artemisinin-based combination treatments have contributed to reductions in the global burden of malaria, prompting the Bill \& Melinda Gates Foundation and the World Health Organization to issue a call in 2007 for an international push towards malaria eradication ${ }^{2}$. Artemisinin drugs normally clear malaria parasites from the blood of a patient within two days of starting treatment; now, however, increasing numbers of Plasmodium falciparum infections in western Cambodia, southern Vietnam, eastern Myanmar and western Thailand take up to five days to clear. In some areas, artemisininbased combination therapies are starting to fail completely, with persistence of both infection and clinical illness after what should be curative treatment.

Efforts to contain artemisinin resistance in southeast Asia and to eliminate malaria would be aided enormously by the identification of a molecular marker for this drug resistance. Such markers are available for resistance to other antimalarial drugs for which the genetic determinants of resistance in the parasite are known. However, neither the mechanism of artemisinin action nor the mechanism(s) of resistance are understood. Examinations of the $P$. falciparum genome for regions of recent strong evolutionary selection, and targeted and genome-wide association studies, have implicated two adjacent regions on chromosome 13 as potential sites of a resistancedetermining gene or genes ${ }^{3,4}$. Through dogged determination and a remarkable combination

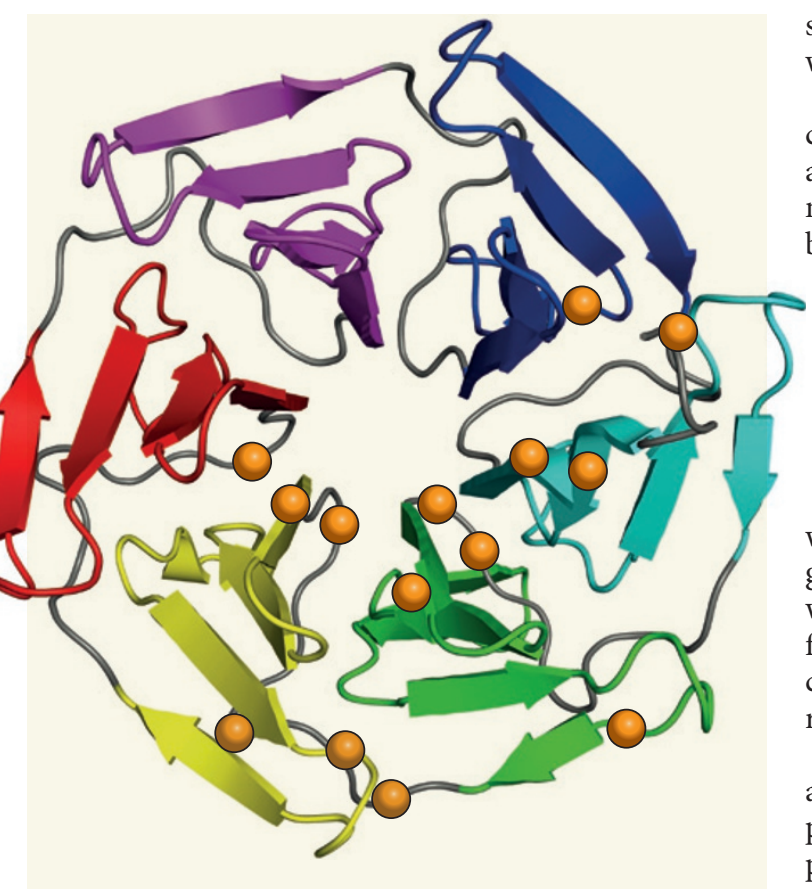

siblings grown in a parallel culture without drug exposure.

The suspects were cornered. The culprit was then identified when the authors looked for these candidate mutations in parasite lines from Cambodia that had varying susceptibilities

to artemisinin drugs in vitro ${ }^{5}$. After ruling out candidate genes from the Tanzanian isolate that showed no sequence variation in the Cambodian isolates, and genes containing SNPs that were not associated with in vitro resistance, a single gene remained with resistance-associated SNPs. The gene is located on chromosome 13, within a candidate region identified in a recent genome-wide association study of clinical artemisinin resistance ${ }^{4}$.

The gene in question encodes a kelch protein called K13. Kelch proteins are involved in a variety of protein-protein interactions, and contain several regions of repeating amino-acid sequences, each forming a 'propeller blade' (Fig. 1). Further evidence for the central role of K13-propeller SNPs in resistance was provided by Ariey and colleagues' ecological survey of malaria parasites from several Cambodian provinces. K13-propeller mutations were rare or absent in sam-

of approaches, Ariey and colleagues seem to have won the race to identify if not the gene, at the very least a key gene, responsible for artemisinin resistance.

In what seemed like a long shot, the researchers laboriously grew an artemisininsensitive parasite isolated from a Tanzanian individual in culture for five years, exposing it intermittently to artemisinin. The drug was removed when the parasites' growth faltered, and replaced after they bounced back. After 60 cycles of drug pressure, the proportion of parasites surviving a pulse of artemisinin had increased from less than $0.01 \%$ to more than $10 \%$. Genome sequencing of this population revealed eight single-nucleotide mutations (single nucleotide polymorphisms, or SNPs) in seven genes that were present in the resistant parasites but not in their ples from provinces with virtually no documented resistance, but were widespread in provinces where resistance has been reported. Moreover, their prevalence in these provinces has increased during the past decade, contemporaneously with increases in the prevalence of resistance.

The authors went on to show that K13 SNPs were also strongly correlated with delayed parasite clearance following artemisinin treatment in clinical trials. And a final piece of evidence came from subpopulations of Cambodian parasites that can be segregated into sensitive and resistant groups ${ }^{6}$. The prevalence of K13 SNPs not only correlated with resistance among these subpopulations, they actually did a better job of explaining resistance than did population groupings: sensitive parasites assigned on the basis of their 
genomic profile to 'resistant' subpopulations had wild-type K13, and resistant parasites belonging to 'sensitive' subpopulations carried the SNPs.

Definitive proof that K13-propeller mutations confer artemisinin resistance will come from genetic transformation of drug-sensitive parasites into resistant ones by the replacement of their wild-type $K 13$ gene with a mutated gene. It is of course possible - even probable - that other genes contribute to artemisinin resistance, but this study leaves little doubt that $\mathrm{K} 13$ is a major determinant of resistance to these drugs in P. falciparum malaria. Further study of gene variants in the chromosomal vicinity of the $K 13$ gene will ascertain whether resistance arose once in western Cambodia and then spread - in which case the genomic regions containing the K13 SNPs will show extended surrounding sequence similarity, indicating common ancestry - or whether it emerged independently in different geographical locales. If resistance has arisen independently in many areas, local containment efforts will be futile and only regional elimination offers any hope of preventing its spread to Africa, where the arrival of drugresistant Asian parasites has previously led to marked increases in malaria hospitalizations and deaths ${ }^{7}$.

Validation of this molecular marker of artemisinin resistance outside Cambodia will be easily achieved, and mapping of the marker throughout southeast Asia is already under way, thanks to early sharing of the results of this study with local malaria-control workers and researchers. With at least 17 SNPs residing in the propeller domains of K13, only one of which is found in any one parasite, sequencing of the $K 13$ gene will initially be necessary to map resistance. But if a few specific SNPs emerge as being predictive of resistance in different settings, switching to rapid molecular assays using DNA extracted from dried blood spots will accelerate translation of this research finding into a practical tool for public-health surveillance.

Christopher V. Plowe is at the Howard Hughes Medical Institute and the Center for Vaccine Development, University of Maryland School of Medicine, Baltimore, Maryland 21201, USA.

e-mail:cplowe@medicine.umaryland.edu

1. Ariey, F. et al. Nature 505, 50-55 (2014).

2. Roberts, L. \& Enserink, M. Science 318, 1544-1545 (2007).

3. Cheeseman, I. H. et al. Science 336, 79-82 (2012).

4. Takala-Harrison, S. et al. Proc. Natl Acad. Sci. USA 110, 240-245 (2013).

5. Witkowski, B. et al. Lancet Infect. Dis. 13, 1043-1049 (2013).

6. Miotto, O. et al. Nature Genet. 45, 648-655 (2013).

7. Trape, J.-F. et al. C.R. Acad. Sci. III 321, 689-697 (1998).

This article was published online on 18 December 2013.

\section{Cloudy with a chance of dustballs}

\section{The flat and featureless transmission spectra of two intermediate-sized extrasolar planets, observed during the planets' passage across their host stars, shed light on the properties of their atmospheres. SEE LETTERS P. 66 \& P.69}

\section{JULIANNE MOSES}

$\mathrm{F}$ lat spectra do not typically excite astronomers, but there are times when a lack of spectral features tells you something interesting. Such is the case with observations of two separate sub-Jupiter-sized extrasolar planets made using the Hubble Space Telescope and reported by Knutson et al. ${ }^{1}$ and Kreidberg et al. ${ }^{2}$ on pages 66 and 69 of this issue, respectively.

The first planet, GJ 436b, has a mass and radius slightly greater than Neptune's. The second, GJ 1214b, is smaller, with a radius roughly 2.7 times that of Earth. Both exoplanets orbit very close to their host stars and are therefore quite warm by Earth standards. Owing to intensive observational scrutiny since their respective discoveries, these two planets have become the archetypes of the new 'Neptuneclass' and 'super-Earth' categories of exoplanets.

Although the first extrasolar planets discovered — in the 1990s and 2000s - tended to be hot, massive, hydrogen-dominated worlds that most closely resemble Jupiter, astronomers have been methodically and efficiently chipping away at the harder-to-observe regime of smaller, denser and cooler Earth-like planets.
Recent ground- and space-based surveys demonstrate ${ }^{3-5}$ that planets of sizes ranging between those of Earth and Neptune overwhelmingly dominate the observed exoplanet population. But what are these intermediate-sized exoplanets really like? Our Solar System does not provide sufficient clues about these planets, because we have only Earth and Venus at one end of the scale and cold Uranus and Neptune at the other to serve as examples. Do these mid-sized exoplanets have rocky surfaces, like Earth and Venus? Are they fluid planets with thick, deep atmospheres relatively rich in hydrogen and volatile elements, like Uranus and Neptune? Are they 'water worlds' with steam atmospheres overlying deep oceans? Are the atmospheres of these planets thick or thin, consisting predominantly of hydrogen, water, carbon dioxide or nitrogen, or something more exotic? Observations of GJ 436b and GJ 1214b provide important hints.

The orbital planes of both GJ 436b and GJ 1214b are almost exactly edge-on as seen from Earth, so that the planets periodically transit - pass directly in front of - their host stars, causing slight dips in the amount of light seen from the system. The depth of these transit dips allows the planetary radius to be determined, and their wavelength dependence provides information about atmospheric composition. During transits, the stellar light passes through the planet's atmosphere on its way to the observer. For a planet with an extended atmosphere, the apparent size of the planet can vary with observed wavelength because more stellar light is blocked at high altitudes at wavelengths for which atmospheric constituents have strong absorption bands. Conversely, more light passes through at low altitudes at wavelengths for which atmospheric constituents are less absorbing.

GJ $1214 \mathrm{~b}$ is known to have a relatively flat (wavelength-independent) transmission spectrum, with weak absorption features at best ${ }^{6,7}$. Because the bulk density of the planet suggests that it must have a gaseous envelope, the two leading theories for explaining the flat transmission spectrum involve either widespread, high-altitude clouds or a hydrogen-poor atmosphere dominated by a high-molecularweight constituent such as water or carbon dioxide (Fig. 1). In both cases, the stellar light at most wavelengths would be extinguished fairly abruptly within a small vertical region of the atmosphere.

In their study, Kreidberg and colleagues present near-infrared transmission spectra for GJ $1214 \mathrm{~b}$ that finally allow one of the two competing theories to be ruled out for this super-Earth exoplanet. The extremely precise spectra, obtained from the Wide Field Camera 3 (WFC3) on board the Hubble Space Telescope, demonstrate that GJ 1214b's transmission spectrum is so flat and featureless in a wavelength region between about 1.1 and 1.6 micrometres that high-altitude clouds provide the only plausible explanation. The observations are precise enough that spectral features from a cloud-free atmosphere dominated by heavy molecules such as water, 